\title{
Effect of computerized cognitive training with virtual spatial navigation task during bed rest immobilization and recovery on vascular function: A pilot study
}

\author{
This article was published in the following Dove Press journal: \\ Clinical Interventions in Aging \\ 10 February 2015 \\ Number of times this article has been viewed
}

\author{
Nandu Goswami' \\ Voyko Kavcic ${ }^{2}$ \\ Uros Marusic ${ }^{3}$ \\ Bostjan Simunic ${ }^{3}$ \\ Andreas Rössler ${ }^{1}$ \\ Helmut Hinghofer-Szalkay' \\ Rado Pisot ${ }^{3}$ \\ 'Institute of Physiology, Medical \\ University of Graz, Graz, Austria; \\ ${ }^{2}$ Institute of Gerontology, Wayne State \\ University, Detroit, MI, USA; ${ }^{3}$ Institute \\ for Kinesiology Research, University \\ of Primorska, Ankaran, Slovenia
}

\begin{abstract}
We investigated the effects of bed rest (BR) immobilization, with and without computerized cognitive training with virtual spatial navigation task (CCT), on vascular endothelium on older subjects. The effects of 14-day BR immobilization in healthy older males $(n=16)$ of ages 53-65 years on endothelial function were studied using EndoPAT ${ }^{\circledR}$, a noninvasive and user-independent method. From the group of 16 older men, 8 randomly received CCT during the $\mathrm{BR}$, using virtual navigation tasks in a virtual environment with joystick device. In all the cases, EndoPAT assessments were done at pre- and post-BR immobilization as well as following 28 days of ambulatory recovery. The EndoPAT index increased from $1.53 \pm 0.09$ (mean \pm standard error of the mean) at baseline to $1.61 \pm 0.16$ following immobilization $(P=0.62)$ in the group with CCT. The EndoPAT index decreased from $2.06 \pm 0.13$ (mean \pm standard error of the mean) at baseline to $1.70 \pm 0.09$ at the last day of BR study, day 14 (BR14) $(P=0.09)$ in the control group. Additionally, there were no statistically significant differences between BR14 and at 28 days of follow-up (rehabilitation program) (R28). Our results show a trend of immobilization in older persons affecting the vasoconstrictory endothelial response. As the control subjects had a greater increase in EndoPAT index after R28 (+0.018) compared to subjects who had cognitive training $(+0.11)$ (calculated from the first day of BR study), it is possible that cognitive training during BR does not improve endothelial function but rather contributes to slowing down the impairment of endothelial function. Finally, our results also show that EndoPAT may be a useful noninvasive tool to assess the vascular reactivity.
\end{abstract}

Keywords: computerized cognitive training, spatial navigation, bed rest, EndoPAT ${ }^{\circledR}$, vascular changes, aging

\section{Introduction}

Bed rest (BR) immobilization is known to occur following falls, accidents, or chronic hospitalization. In addition, elderly populations are frequently confined to BR. Similarly, BR immobilization is frequently used in ground-analog to simulate the physiological changes induced in astronauts during their prolonged stay in low-earth orbit such as in the International Space Station.,

The endothelium plays an important role in the regulation of vasodilation, vascular inflammation, and coagulation. ${ }^{3}$ Similar to other cardiovascular risk factors, ${ }^{4}$ aging is associated with altered endothelial function and signaling. ${ }^{5}$ We have recently reported that endothelial function is altered following 20 min of standing. ${ }^{6}$ To our knowledge, we are not aware of any study that has examined how the endothelium is
Correspondence: Voyko Kavcic Institute of Gerontology, Wayne State University, 87 E. Ferry Street,

Detroit, MI 48202, USA

$\mathrm{Tel}+\mathrm{I} 3136642613$

$\mathrm{Fax}+\mathrm{I} 3136642667$

Email voyko@wayne.edu 
disturbed under conditions of gravitational unloading. This is particularly important, as alterations of endothelial cells, which play important roles in vasoconstriction, thrombosis, and inflammation, are known to occur early in the pathogenesis of atherosclerosis. As the vascular endothelium affects the regulation of vascular tone, ${ }^{7}$ it may play a role in the pathophysiology of orthostatic intolerance. Up to date, however, there have been limited studies on the impact of BR on the vascular endothelium.

\section{Cognitive training}

In the context of the rapid increase in longevity, ubiquitous age-related declines in cognitive function have important socioeconomic implications. Thus, understanding mechanisms leading to these declines is vital for the development of environmental and clinical interventions geared toward cognitive rehabilitation of older adults. An important nonpharmacological approach that could be used for maintaining and/or preventing decline of cognitive capacities in older age is use of cognitive training. Cognitive training generally involves guided practice of standard tasks to increase or maintain particular cognitive functions such as memory. These training tasks intend to challenge cognitive abilities and induce learning. A variety of tasks and approaches have been used for cognitive training (for review, see Tardif and Simard), ${ }^{8}$ and most of the studies reported significant changes in performance on cognitive function training. ${ }^{9,10}$

The goal of this study is to further our understanding of the fundamental adaptive homeostatic mechanisms involved in changes in endothelial function in bed-rested older subjects, with and without cognitive training. As mechanical stress of the endothelium has been associated with several substances that act on vessel diameter, ${ }^{10-12}$ and we have shown that an orthostatic challenge of 20 min (active standing) provides the shear stress and affects vascular function, ${ }^{6}$ we investigated the vascular changes that occur in bed-rested subjects (ie, without orthostatic loading). As the available literature suggests that orthostasis increases flow-mediated dilatation (FMD), ${ }^{13,14}$ we hypothesized that in bed-rested subjects there will be a reduction in the FMD (hypothesis \#1). As mental challenge is known to cause vasodilation in the skeletal muscles, ${ }^{15}$ we also hypothesized that cognitive training in the older subjects would prevent this reduction in the FMD that occurs during BR immobilization (hypothesis \#2). As the effects of cognitive training are present for long periods following the training, we tested the hypothesis that the cognitive effects would continue during recovery (hypothesis \#3).

\section{EndoPAT $^{\circledR}$}

Brachial artery FMD is normally used to assess endothelial changes. However, it is highly operator dependent and requires special methodological and physiological considerations. ${ }^{16}$ This study, therefore, utilized the EndoPAT ${ }^{\circledR}$, a noninvasive method of assessing endothelial function in $15 \mathrm{~min}$. While brachial ultrasound assessments depend on an ultrasonologist, EndoPAT measurements are independent of the person doing the measurements. It depends on peripheral arterial tone (PAT) signal, ${ }^{17}$ which is measured from the fingertip by measuring arterial pulsatile volume changes. The EndoPAT supports a pair of modified plethysmographic biosensors ${ }^{18}$ that apply a uniform sub-diastolic pressure field to the distal end of the fingers, thus preventing distal venous pooling, which can cause a veno-arterial vasoconstrictor reflex. The finger probes are connected by flexible tubing to isolated volume reservoirs, ${ }^{19}$ which are then connected to a computer.

\section{EndoPAT testing protocol}

Testing is carried out with the volunteer in supine position. The hand is elevated so the fingers hang freely without touching any surface. Two small biosensors are placed on both the index fingers. A standard blood pressure cuff is placed on one arm. During testing, occlusion of the brachial artery - to cause stoppage of blood flow to the arm - is achieved by inflating the cuff to suprasystolic pressures for 5 min. ${ }^{17,19}$ When the cuff is released, the surge of blood flow causes endothelial-dependent FMD, which is manifested as reactive hyperemia, and is shown as increases in the PAT signal amplitude. The inbuilt EndoPAT software calculates the postocclusion to preocclusion ratio (EndoPAT index). In high responders pulse amplitude increases but not in subjects with compromised endothelial function. ${ }^{17}$

\section{Methodology Subjects}

Twenty-three males, 16 older adults aged between 53 and 65 and 7 younger adults aged between 19 and 28 volunteered to participate in the project "Bed Rest Study - PANGeA, Valdoltra 2012 - The effects of simulated weightlessness on the human organism". For the purposes of the present study, a total of 16 healthy, untrained, older male subjects (59.6 \pm 0.8 years, mean \pm standard error of the mean) were considered. They were recruited through public promotions of the project, newspaper advertisements, and wordof-mouth recommendation from three coastal towns in Slovenia. Details on age and body mass index can be found in Table 1. 
Table I Age, BMI and BP of the older subjects from the CT group (with CT) and control groups (without CT), at BRI, BR I4, and R28

\begin{tabular}{|c|c|c|c|c|}
\hline \multirow[t]{3}{*}{ Measure } & \multirow{2}{*}{\multicolumn{2}{|c|}{$\begin{array}{l}\text { Older }(53-65 \text { years }) \\
\text { CT group }(\mathrm{N}=8)\end{array}$}} & \multirow{2}{*}{\multicolumn{2}{|c|}{$\frac{\text { Older }(56-65 \text { years })}{\text { Control group }(\mathrm{N}=8)}$}} \\
\hline & & & & \\
\hline & Mean & SEM & Mean & SEM \\
\hline Age (years) & 59.4 & 1.3 & 59.9 & 1.2 \\
\hline BMI BRI (kg/m²) & 26.1 & 1.8 & 26.6 & 1.4 \\
\hline BMI BRI4 (kg/m²) & 25.3 & 1.6 & 25.8 & 1.4 \\
\hline BMI R28 $\left(\mathrm{kg} / \mathrm{m}^{2}\right)$ & 26.2 & 1.7 & 26.5 & 1.5 \\
\hline BP systolic/diastolic BRI (mmHg) & II5.6/68.1 & $5.0 / 3.3$ & $118.8 / 80.6$ & $5.8 / 5.4$ \\
\hline BP systolic/diastolic BR I4 (mmHg) & I23.8/79.4 & $3.8 / 2.7$ & $120.6 / 80.0$ & $3.7 / 2.5$ \\
\hline
\end{tabular}

Abbreviations: BMI, body mass index; BP, blood pressure; BR, bed rest; CT, cognitive training; BRI, first day of BR study; BRI4, last day of BR study, day I4; R28, at 28 days of follow-up (rehabilitation program); SEM, standard error of the mean.

All subjects were right-handed, had normal or correctedto-normal vision, reported no history of cardiovascular disease, neurological or psychiatric conditions, vertigo, vestibular problems, or problems with gait or balance, and had no histories of drug/alcohol abuse.

Subjects were familiarized with the equipment, measurements, and personnel. Subjects were tested between $2 \mathrm{pm}$ and $4 \mathrm{pm}$. Care was taken to ensure the subjects were tested at the same time of the day for subsequent measurements. During supine BR, participants were allowed to only turn to their sides, put no more than two pillows under the head, and were not allowed to stand up, sit on the bed, or raise the arms above their heads.

The tests were carried out in a quiet room, maintained at $22^{\circ} \mathrm{C}-23^{\circ} \mathrm{C}$ and humidity at $50 \%-55 \%$, at the Valdoltra Hospital, University of Primorska, Ankaran, Slovenia. All procedures were carried out in accordance with the Declaration of Helsinki and were approved by the Republic of Slovenia National Medical Ethics Committee. Verbal and written informed consent was obtained from all subjects prior to the BR experiment. Signed informed consent forms were then stored at the University of Primorska, Slovenia.

\section{Study design}

We divided our subjects randomly in two groups: eight supine bed-rested older subjects who on each day performed $50 \mathrm{~min}$ of spatial navigation training in a virtual environment (VE) with joystick (CT group) and another eight supine bed-rested older subjects who watched documentaries on Discovery channel for $50 \mathrm{~min}$ (control group) in a separate room.

The BR was a controlled longitudinal interventional study, which lasted 14 days, followed by 28 days of recovery period. The study was a mixed $2 \times 3$ design with group $(\mathrm{CT}$, control groups) as a between-subject variable and time (pretest/posttest 1/posttest 2) as within-subject variable. Pretest was done on the first day of BR study (BR1) and posttest 1 was administered on the last day of BR study, day 14
(BR14). Posttest 2 was administered after conclusion of at rehabilitation program at 28 days of follow-up (rehabilitation program) (R28) primarily to assess: (1) the effectiveness of rehabilitation program, ie, how fast the measurements that were affected by BR returned to baseline values and (2) whether there was any continued effect of the cognitive training upon completion of the rehabilitation.

\section{Spatial navigation training}

All VEs were designed using modified version of Unreal Tournament 2003 and the Unreal Editor 3.0 (Epic Games, Inc) software package. During periods of cognitive training, all the subjects were lying in bed and controlled their movements within the VEs using a Trust Predator Joystick GM-2550. The tasks were presented on a 17-inch flat-panel LCD monitor situated approximately $60 \mathrm{~cm}$ in front of the subjects.

To ensure that all subjects were equally adept at controlling movement to the point of navigating at high speed and making quick turns in the VEs prior to cognitive training in mazes, the subjects were given joystick training. It involved navigating through an environment consisting of a corridor with several turns and that was equal in width to those seen in the training mazes (512 virtual units). All subjects completed the joystick-training environment under the required time of $120 \mathrm{sec}$. After completing training, subjects were instructed to solve each intersection maze by proceeding along the correct path to a goal area with a trophy in it as quickly and with as few errors as possible. This was done for five trials in total.

CT group of subjects was specifically asked to navigate through VEs with use of a joystick device on each day of BR study for approximately $50 \mathrm{~min}$. Upon successful joystick training, subjects were given cognitive training in several maze VEs of increasing difficulty. All mazes consisted of a series of interconnected corridors, with three available paths at each intersection or decision point. A pair of verbal or nonverbal cues was displayed in the VE at each decision 
point, placed at either opposite corner of the intersection, and in corridors at various nondecision points. Verbal cues consisted of signs with country names, city names, and animal names. Nonverbal cues consisted of country flags, animal pictures, and human faces. Subjects were instructed to select the correct path leading to the goal area as quickly and efficiently as possible.

In each maze, time to completion, distance traveled, average speed, number of errors (turns to incorrect corridors at a decision point), and number of backtracks (returns to previously navigated areas) were recorded automatically by Unreal Navigation UT software. Subjects were trained in mazes with three intersections, five intersections, and seven intersections. Subjects were to complete six three-intersection mazes, followed by 6 five-intersection mazes, and 12 seven-intersection mazes. Although the number of seven-intersection mazes completed by the end of the training period varied depending on how quickly each subject reached criterion on each maze, all subjects who went through cognitive training completed at least six of the seven-intersection mazes.

\section{EndoPAT measurements}

Each subject had an initial (pre) blood pressure measurement made when supine. The recorded individual systolic blood pressure values were used to calculate for each subject the suprasystolic values ( $20 \mathrm{mmHg}$ above the systolic pressure) to which the sphygmomanometer cuff was inflated. After blood pressure measurements, no measurements were done for $20 \mathrm{~min}$. Then the endothelial function was assessed using EndoPAT2000 ${ }^{\circledR}$ (Itamar Medical Ltd, Caesarea, Israel, see Cvirn et $\mathrm{al}^{3}$ ). Each test was carried out with the subject in the supine position. The hands were elevated so the fingers were hanging freely without touching any surface, and two plethysmographic biosensors (finger probes) (Itamar Medical Ltd) were applied to each index finger. The fingers were allowed to hang freely off a small platform. A standard blood pressure cuff (DS 400 Aneroid Sphygmomanometer, Hokanson, Bellevue, WA, USA) was placed around the left arm. The finger probes were attached to the EndoPAT2000 by means of flexible tubing to volume reservoirs. The EndoPAT2000 was connected to a computer with EndoPAT software (see Cvirn et $\mathrm{al}^{3}$ ).

Following 5 min PAT signal measurement (baseline), the blood pressure cuff was inflated up to $20 \mathrm{mmHg}$ greater than each subject's systolic blood pressure values (suprasystolic pressure), thus occluding the forearm blood flow. As the software allows for real-time viewing of the PAT signal, suprasystolic pressure could be confirmed by seeing a flat line instead of the pulsatile PAT signal seen in the control arm. The cuff stayed inflated for a period of 5 min following which the cuff pressure was released. The PAT signal was measured for a further 5 min period during recovery.

The EndoPAT software allows for real-time viewing of the PAT signal and for subsequent review and automatic analysis to give an EndoPAT/Reactive Hyperemia Index, also called the Endoscore. The Reactive Hyperemia Index represents a ratio of the average pulse wave amplitude during a time frame of $1 \mathrm{~min}, 60 \mathrm{sec}$ after the release of pressure from the blood pressure cuff, to the average pulse wave amplitude of a $210 \mathrm{sec}$ baseline period prior to the blood pressure cuff being inflated. This index takes into account the PAT signal from both the finger on the arm where occlusion has taken place, as well as the contralateral "control" finger. In doing this, the index corrects for any systemic vascular changes that may be taking place due to occlusion to arterial flow in the measurement arm.

\section{Treatment of EndoPAT data}

For each subject, the EndoPAT index was recorded at the start of the study (baseline), at the end of the BR (BR14), and after 28 days of recovery (recovery: R28). The effects of cognitive training on EndoPAT measurements were evaluated by using one-way repeated-measures analysis of variance with time (baseline, BR14, and recovery) as within-subject variable. We also used within-subject contrast as a primarily linear and quadratic term, to detect significant changes in successive measurements. The effects of BR were confirmed by significant linear and/or quadratic term. Statistical analysis was performed by SPSS (SPSS, Inc., Chicago, IL, USA).

\section{Rehabilitation protocol}

Rehabilitation program lasted 4 weeks. It started on the 2 nd day post-BR and finished on the 28th day. It consisted of 10 sessions performed three times a week for approximately 65 min with 24-48 hours of rest between sessions. Each session consisted of a warm-up protocol, 10 functional balance and strength/power exercises, an endurance interval training protocol, and some relaxation exercises at the end of the session. Six coaches (leaders), each of whom had to guide up to four participants throughout the program, conducted the rehabilitation/training program. The coaches were experienced students from a local Applied Kinesiology study program as well as members of the research team who were well trained and familiarized with the training program before the start.

The warm-up protocol lasted approximately $12 \mathrm{~min}$ and included 6 min of Nordic walking (intensity at the speed determined from the pre-BR $2 \mathrm{~km}$ walk test) and $6 \mathrm{~min}$ of 
10 active stretching exercises and two activation exercises. Participants performed one set of eight repetitions of active stretching and activation exercises before starting the functional balance and strength/power training.

The first four exercises for improving balance and strength/power were chosen so that balance and strength requirements were combined together (balance/strength exercises). The other six exercises were on the other hand focused only on strength and later, with participants' improvement, on power (strength/power exercises). All exercises had one or two modifications for increasing their difficulty throughout the training period. The balance/strength exercises were modified on sessions $3-5$ and sessions $6-10$, and the strength/ power exercises were modified on sessions 5-10. The difficulty and/or intensity of the exercises were controlled for each subject by the coaches. For the first four exercises, the participants had to pay attention on active establishing (not maintaining) of the balance during the execution without falling and with as little help as possible. For the other six exercises the participants were instructed to choose the highest possible weight to perform 10-12 repetitions during sessions $1-5$. However, during sessions $6-10$, they had to perform the exercises as fast as possible with the maximum possible weight to perform 15 repetitions. This protocol was organized as circuit training, comprising 1 min cycles, and lasted approximately $20 \mathrm{~min}$.

After completing the balance/strength/power exercise protocol, the participants also performed an endurance protocol, which consisted of Nordic walking. It was organized in 30 to 45 sec intervals. During the first two or three sessions, teaching as well as emphasis on proper technique of Nordic walking was a priority. In this period, for the given time interval, the distance to be achieved by the participants was individually defined, based on the walking speed determined from the pre-BR $2 \mathrm{~km}$ walk test. From the fourth session on, each participant was instructed - and then encouraged - during walking to be as fast as possible without affecting the walking technique. The whole protocol lasted approximately 20-30 min.

In the final part of each session, which lasted 3-5 min, the participants tried to relax as much as possible doing some breathing exercises and few movements of the legs and arms.

\section{Results}

The EndoPAT index for both groups at BR1, BR14, and R28 are presented in Table 2 .

\section{The effect of BR on endothelial function (hypothesis \#I)}

The effects of BR immobilization on EndoPAT index were evaluated by comparing EndoPAT differences from beginning and end of BR measurements. The EndoPAT index increased from $1.53 \pm 0.09$ (mean \pm standard error of the mean) at baseline to $1.61 \pm 0.16$ following immobilization $(P=0.62)$ in the $\mathrm{CT}$ group. The EndoPAT index decreased from 2.06 \pm 0.13 (mean \pm standard error of the mean) at baseline to $1.70 \pm 0.09$ following immobilization $(P=0.09)$ in the control group. That is, we did not observe any significant change in the CT group, and it did not reach the level of significance in the control group.

\section{Effect of cognitive training on endothelial function during immobilization (hypothesis \#2)}

We evaluated effects of cognitive training on EndoPAT separately for each group by entering EndoPAT index into one-way repeated-measures analysis of variance with time (BR1, BR14, and R28) as a within-subject variable. There was no significant change of reactive hyperemia with time in patients with cognitive training $(P=0.44)$, while a transitory drop in this parameter in controls was found as shown by EndoPAT indexes at BR1=2.06, at BR14=1.70, and at $\mathrm{R} 28=2.22(P=0.02)$.

\section{Recovery responses across the groups (hypothesis \#3)}

In this study, we also examined the effect of BR and the recovery phases on the endothelial function. This was done by comparing endothelial functions at the end of BR (BR14) and recovery (R28) to baseline values. In both groups, there

Table 2 EndoPAT measurements across the groups

\begin{tabular}{|c|c|c|c|c|c|c|c|}
\hline & \multirow[t]{2}{*}{ Groups } & \multicolumn{2}{|l|}{ BRI } & \multicolumn{2}{|l|}{ BR I 4} & \multicolumn{2}{|l|}{$\mathbf{R} 28$} \\
\hline & & Mean & SEM & Mean & SEM & Mean & SEM \\
\hline \multirow[t]{2}{*}{ EndoPAT index } & Cognitive training $(\mathrm{N}=8)$ & 1.53 & 0.09 & 1.61 & 0.16 & 1.64 & 0.14 \\
\hline & Control $(\mathrm{N}=8)$ & 2.06 & 0.13 & 1.7 & 0.09 & 2.22 & 0.14 \\
\hline
\end{tabular}

Abbreviations: BR, bed rest; BRI, first day of BR study; BRI4, last day of BR study, day I4; R28, at 28 days of follow-up (rehabilitation program); SEM, standard error of the mean. 
was increased level of EndoPAT index at R28 as compared to BR1. In the CT group, there was an increase of 0.11 (BR1=1.53, R28=1.64, $P=0.21$ ), and in controls, there was an increase of $0.16(\mathrm{BR} 1=2.06, \mathrm{R} 28=2.22, P=0.12)$. Both these were not significant. In addition, there were no statistically significant differences between BR14 and R28.

\section{Discussion}

Our results, obtained using the noninvasive, user-independent EndoPAT, show a trend of immobilization affecting the vasoconstrictory endothelial response as well as modulation of the endothelial response by cognitive challenge. To our knowledge, this is first report showing that: (1) BR immobilization seems to affect EndoPAT values in older subjects and (2) cognitive training also shows a trend toward preventing a decline in peripheral vascular function during immobilization/ prolonged inactivity. Our results in older subjects suggest that cognitive challenge during immobilization might be sufficient to prevent BR-induced vascular (pathological) effects. Considering the role vascular endothelium plays in thrombosis, post-BR orthostatic intolerance, and cardiovascular diseases, our results have application in coagulation research and prevention of cardiovascular accidents as well as geriatrics.

We speculate that the beneficial effects of cognitive training on vascular function in immobilized subjects arises via the following mechanisms:

1. Cognitive tasks, similar to mental challenging, are known to activate the sympathetic activity. Increases in sympathetic activity induced by cognitive tasks cause increases in heart rate and cardiac output thus leading to increases in mean arterial pressure. As cardiac output represents global tissue perfusion, at least in healthy subjects, it is possible that the application of cognitive activity leads to increased blood flow in different regions such as the brain and muscles, leading to increases in tissue perfusion and prevention of muscle atrophy, which would otherwise occur in immobilization. This hypothesis is supported by the observations of Kuipers et $\mathrm{al}^{15}$ who reported that during mental challenge there is vasodilation in the skeletal muscle.

In addition, mental challenge also affects an important reservoir of the blood in the body: the venous system. As the venous system is an important capacitance area of the body, alterations in its capacity can cause large changes in venous return and cardiac preload. It is possible that the increases in sympathetic activity that accompany mental challenge could have increased the cardiac output via greater increases in venous tone and increases in mean circulatory filling pressure, thus leading to improved venous return (see Guyton's analysis). ${ }^{20}$

We have also previously reported that mental challenge-induced increases in sympathetic activity could lead to improvements in orthostatic tolerance times in subjects undergoing graded lower body negative pressure until presyncope. ${ }^{21}$

2. It is possible that cortical activation induced by the cognitive activity could have led to the modification of reflex regulation of blood pressure, via its effects on the baroreflexes. ${ }^{22}$

3. Another possible mechanism is that cognitive training could have activated brain structures involved in mobility and walking, which, in turn, could affect peripheral blood flow. For example, imaging studies have shown that the same premotor cortex and supplementary motor areas that are involved in real walking are activated during mental imagery of movement. ${ }^{23,24}$ Indeed, both observations of walking and virtual walking produce similar, and even higher, levels of cerebral activation compared to real walking. ${ }^{25,26}$ These findings suggest that our cognitive training emphasizing virtual movement may activate the same neural systems involved in mobility. In turn, activation of cerebral structures involved in the walking may to the same degree also generate subliminal or even supraliminal muscle contractions/activation, which in turn may affect blood flow to the muscles.

A limitation of our study is the sample size. This is mainly due to ethical limitations, which restricted the number and age of potential subjects. In addition, the differences between the two groups at the zero time point (BR1) and 4 weeks after the BR (R28) appears much larger than the effect of prolonged $\mathrm{BR}$ or the cognitive training. That difference could have arisen due to a sampling/randomization problem. However, we do not believe that to be the case as the patients were not aware to which group they would belong to on day 1 of the BR (BR1) measurement.

\section{Conclusion}

Our results show a trend of immobilization in older subjects affecting the vasoconstrictory endothelial response. As the control subjects had a greater increase in EndoPAT index after $\mathrm{R} 28(+0.018)$ compared to subjects who had cognitive training $(+0.11)$ (calculated from BR1), it is possible that cognitive training during BR does not improve endothelial function but rather contributes to slowing down the impairment of endothelial function. Finally, our results also show that EndoPAT may be a useful noninvasive tool to assess the vascular reactivity. 


\section{Acknowledgments}

We would like to thank the participants in the study for their time and effort to ensure the success of the project. We acknowledge the excellent assistance of the entire staff of the Valdoltra Orthopaedic Hospital of Ankaran (Koper, Slovenia). Additionally, we thank the research team and the students of Applied Kinesiology of University of Primorska for the help and logistic support and many others who contributed to the smooth undertaking of the study. The study was conducted by Slovenian and Italian partners in the framework of the standard project PANGeA: Physical Activity and Nutrition for Quality Ageing, supported by a grant from the Crossborder Cooperation Program Slovenia - Italy 2007-2013 and co-financed by the European Regional Development Fund as well as the Slovenian and Italian national funds.

\section{Disclosure}

The authors report no conflicts of interest in this work. The funders had no role in the study design, data collection and analysis, decision to publish, or preparation of the manuscript.

\section{References}

1. Goswami N, Batzel JJ, Clément G, et al. Space physiology I: maximizing information from space data resources: a case for expanding integration across research disciplines. Eur J Appl Physiol. 2013;113(7):1645-1654.

2. Marusic U, Meeusen R, Pisot R, Kavcic V. The brain in micro- and hypergravity: the effects of changing gravity on the brain electrocortical activity. Eur J Sport Sci. 2014;38:1-10.

3. Cvirn G, Schlagenhauf A, Leschnik B, et al. Coagulation changes during presyncope and recovery. PLoS One. 2012;7(8):e42221-e42221.

4. Bondarenko A, Panasiuk O, Stepanenko L, Goswami N, Sagach V. Reduced hyperpolarization of endothelial cells following high dietary $\mathrm{Na}^{+}$: effects of enalapril and tempol. Clin Exp Pharmacol Physiol. 2012;39:608-613.

5. Sagach V, Bondarenko A, Bazilyuk O, Kotsuruba A. Endothelial dysfunction: possible mechanisms and ways of correction. Exp Clin Cardiol. 2006; $11: 107-110$

6. Goswami N, Gorur P, Pilsl U, et al. Effect of orthostasis on endothelial function: a gender comparative study. PLoS One. 2013;8(8):e71655.

7. Levick JR. An Introduction to Cardiovascular Physiology. 5th ed. Abingdon, OX: Hodder Education; 2012.

8. Tardif S, Simard M. Cognitive stimulation programs in healthy elderly: a review. Int J Alzheimers Dis. 2011;2011:378934.

9. Ball K, Berch DB, Helmers KF, et al; Advanced Cognitive Training for Independent and Vital Elderly Study Group. Effects of cognitive training interventions with older adults: a randomized controlled trial. JAMA. 2002;288:2271-2281

Clinical Interventions in Aging

\section{Publish your work in this journal}

Clinical Interventions in Aging is an international, peer-reviewed journal focusing on evidence-based reports on the value or lack thereof of treatments intended to prevent or delay the onset of maladaptive correlates of aging in human beings. This journal is indexed on PubMed Central, MedLine,
10. Willis SL, Tennstedt SL, Marsiske M. Long-term effects of cognitive training on everyday functional outcomes in older adults. JAMA. 2006; 296:2805-2814.

11. Furchgott RF, Zawadzki JV. The obligatory role of endothelial cells in the relaxation of arterial smooth muscle by acetylcholine. Nature. 1980;288:373-376.

12. Münzel T, Sinning C, Post F, Warnholtz A, Schulz E. Pathophysiology, diagnosis and prognostic implications of endothelial dysfunction. Ann Med. 2008;40:180-196.

13. Guazzi M, Lenatti L, Tumminello G, Guazzi MD. Effects of orthostatic stress on forearm endothelial function in normal subjects and in patients with hypertension, diabetes, or both diseases. Am J Hypertens. 2005;18:986-994.

14. Guazzi M, Lenatti L, Tumminello G, Puppa S, Fiorentini C, Guazzi MD The behaviour of the flow-mediated brachial artery vasodilatation during orthostatic stress in normal man. Acta Physiol Scand. 2004;182:353-360.

15. Kuipers NT, Sauder CL, Carter JR, Ray CA. Neurovascular responses to mental stress in the supine and upright postures. J Appl Physiol. 2008;104:1129-1136.

16. Thijssen DH, Black MA, Pyke KE, et al. Assessment of flow-mediated dilation in humans: a methodological and physiological guideline. Am J Physiol Heart Circ Physiol. 2011;300:H2-H12.

17. Hamburg NM, Benjamin EJ. Assessment of endothelial function using digital pulse amplitude tonometry. Trends Cardiovasc Med. 2009; 19:6-11.

18. Kuvin JT, Patel AR, Sliney KA, et al. Assessment of peripheral vascular endothelial function with finger arterial pulse wave amplitude. Am Heart J. 2003;146:168-174.

19. Faizi AK, Kornmo DW, Agewall S. Evaluation of endothelial function using finger plethysmography. Clin Physiol Funct Imaging. 2009; 29:372-375

20. Montani JP, Van Vliet BN. Understanding the contribution of Guyton's large circulatory model to long-term control of arterial pressure. Exp Physiol. 2009;94:382-388.

21. Goswami N, Roessler A, Hinghofer-Szalkay H, Montani JP, Steptoe A. Delaying orthostatic syncope with mental challenge: a pilot study. Physiol Behav. 2012;106:569-573.

22. Stephenson RB. Modification of reflex regulation of blood pressure by behavior. Ann Rev Physiol. 1984;46:133-142.

23. Iseki K, Hanakawa T, Shinozaki J, Nankaku M, Fukuyama H. Neural mechanisms involved in mental imagery and observation of gait. $\mathrm{Neu}$ roimage. 2008;41(3):1021-1031.

24. Wagner J, Stephan T, Kalla R, et al. Mind the bend: cerebral activations associated with mental imagery of walking along a curved path. Exp Brain Res. 2008;191(2):247-255.

25. Kranczioch C, Mathews C, Dean P, Sterr A. Task complexity differentially affects executed and imagined movement preparation: evidence from movement-related potentials. PLoS One. 2010;5(2):e9284.

26. Yinlai J, Wang S, Tan R, et al. Comparison of cortical activation during real walking and mental imagery of walking - The possibility of quickening walking rehabilitation by mental imaginary of walking. In: Theophanides T, editor. Infrared Spectroscopy - Life and Biomedical Sciences. Rijeka: InTech; 2012:378. [ISBN: 978-953-51-0538-1].

CAS, Scopus and the Elsevier Bibliographic databases. The manuscript management system is completely online and includes a very quick and fair peer-review system, which is all easy to use. Visit http://www.dovepress. com/testimonials.php to read real quotes from published authors. 